\title{
n-3 Polyunsaturated Fatty Acids Decrease Long-Term Diabetic Risk of Offspring of Gestational Diabetes Rats by Postponing Shortening of Hepatic Telomeres and Modulating Liver Metabolism
}

\author{
Jinlong Gao ${ }^{1}$, Hailong Xiao ${ }^{2}$, Jiaomei Li ${ }^{3}$, Xiaofei Guo ${ }^{3}$, Wenwen Cai ${ }^{1}$ and Duo Li ${ }^{1,3, *(\mathbb{D})}$ \\ 1 Department of Food Science and Nutrition, Zhejiang University, 866 Yuhangtang Road, \\ Hangzhou 310058, China \\ 2 Hangzhou Institute for Food and Drug Control, 198 Yonghua Street, Hangzhou 310022, China \\ 3 Institute of Nutrition and Health, Qingdao University, 308 Ningxia Road, Qingdao 266071, China \\ * Correspondence: duoli@zju.edu.cn or duoli@qdu.edu.cn; Tel.: +86-532-8299-1018
}

Received: 17 May 2019; Accepted: 19 July 2019; Published: 23 July 2019

\begin{abstract}
The long-term influence of gestational diabetes mellitus (GDM) on offspring and the effect of omega-3 polyunsaturated fatty acids ( $n-3$ PUFA) on GDM offspring are poorly understood. We studied the long-term diabetic risk in GDM offspring and evaluated the effect of $n-3$ PUFA intervention. Healthy offspring rats were fed standard diet (soybean oil) after weaning. GDM offspring were divided into three groups: GDM offspring (soybean oil), $n-3$ PUFA adequate offspring (fish oil), and $n$-3 PUFA deficient offspring (safflower oil), fed up to 11 months old. The diabetic risk of GDM offspring gradually increased from no change at weaning to obvious impaired glucose and insulin tolerance at 11 months old. $n-3$ PUFA decreased oxidative stress and inflammation in the liver of older GDM offspring. There was a differential effect of $n-3$ PUFA and $n-6$ PUFA on hepatic telomere length in GDM offspring. Non-targeted metabolomics showed that $n-3$ PUFA played a modulating role in the liver, in which numerous metabolites and metabolic pathways were altered when GDM offspring grew to old age. Many metabolites were related to diabetes risk, such as $\alpha$-linolenic acid, palmitic acid, ceramide, oxaloacetic acid, tocotrienol, tetrahydro-11-deoxycortisol, andniacinamide. In summary, GDM offspring exhibited obvious diabetes risk at old age, whereas $n-3$ PUFA decreased this risk.
\end{abstract}

Keywords: gestational diabetes mellitus; offspring; long-term effect; $\mathrm{n}-3$ polyunsaturated fatty acid; diabetes risk; liver; oxidative stress; inflammation; telomere length; metabolomics

\section{Introduction}

Gestational diabetes mellitus (GDM) increases the offspring's risk of developing obesity, diabetes, cardiovascular disease, and other diseases [1]. The long-term impact of GDM on offspring, however, is poorly understood. The risk and mechanisms of developing diabetes during GDM offspring growth, especially as they grow to an old age, are not well known. N-3 polyunsaturated fatty acids (PUFA) play a role in preventing cardiovascular disease, hypertension, cancer, and cognitive decline [2]. Nevertheless, the relationship between $n-3$ PUFA and diabetes risk varies from study to study, even with opposite conclusions [3,4]. Furthermore, it remains unclear whether $n-3$ PUFA can decrease diabetic risk for GDM offspring. Several studies have suggested possible mechanismsfor GDM offspringto develop diabetes, including an abnormal insulin signaling pathway, lower antioxidant capacity, lipid metabolism disorder, and epigenetic alterations [5-8]. However, other mechanisms need to be studied to prevent the diabetic occurrence in GDM offspring. 
Telomeres, which are termini of chromosomes, contain specific DNA sequences. Telomeres are essential for chromosomal integrity. The length of telomeres shortens with age [9]. Accelerated telomere shortening is caused by some adverse factors such as oxidative stress and inflammation [10]. Shortening of telomere length is a biomarker of aging, and it is linked to diseases risk [11]. Metabolomics plays a role in studying metabolic changes. Several studies based on metabolomics have focused on GDM mothers, investigating the correlation between abnormal metabolites and GDM or finding biomarkers that can predict GDM risk [12-14]. Only a few studies have analyzed metabolic changes of GDM offspring at birth and infancy $[15,16]$. Nevertheless, long-term effects of GDM on metabolic alterations in offspring are not well known.

The liver is the main organ for the regulation of blood glucose [17]. Theory of developmental origins of health and disease ( $\mathrm{DOHaD}$ theory) and fetal programming hypothesis reveal that the risk of adult diseases later in life is associated with adverse intrauterine conditions $[18,19]$. GDM is a typical adverse intrauterine condition in which placental transport of nutrients to the fetus is altered and some adverse factors such as oxidative stress and inflammation cause damage to the fetal liver and other organs [20]. First, aging is an inevitable process. This lets us question whether GDM can cause hepatic aging of offspring later in life and whether the length of hepatic telomeres in GDM offspring will be obviously shortened at old age. Furthermore, the effect of $n-3$ PUFA and $n-6$ PUFA on the length of hepatic telomeres in GDM offspring remains unclear. Second, the liver is the center of metabolism. This allow us to consider whether GDM can lead to long-term metabolic changes of the liver of offspring, increasing the risk of developing diabetes at old age. Moreover, the long-term effect of $n-3$ PUFA and $n-6$ PUFA on hepatic metabolism of GDM offspring has not been studied.

The aim of this study was to investigate the risk of developing diabetes during GDM offspring rats' growth, particularly when they grow to 11 months old. Meanwhile, the effect of $n-3$ PUFA on diabetes risk of GDM offspring was studied. For the first time, this study discovered the differential effect of $n-3$ PUFA and n-6 PUFA on the length of hepatic telomeres in GDM offspring. Moreover, metabolomics analysis revealed obvious metabolic alterations of the liver of GDM offspring at an old age. $n-3$ PUFA played an important role in modulating the metabolism of the liver of GDM offspring.

\section{Materials and Methods}

\subsection{Animal Model and Offspring Diet Intervention}

The animal protocols were approved by Zhejiang University Animal Center and Ethic Committee. The protocol of the study is shown in Figure 1. Forty-four female 10-week-old virgin Wistar female rats and 22 male adult rats were obtained and fed purified AIN-93 diet. The diet composition was as follows (g/kg): starch, 397; maltodextrin, 132; casein, 200; sucrose, 100; soybean oil, 70; fiber, 50; mineral mix, 35; vitamin mix 10; L-cystine, 3; choline bitartrate, 2.5; Tert-butylhydroquinone, 0.14 . After one week of acclimation, female rats were randomly divided into two groups: healthy maternal group $(n=10)$ and GDM group $(n=34)$. Female rats, two per cage, mated with male rats overnight by ratio of 2:1. Day 0 of gestation was confirmed by presence of sperm in vaginal smears. On the fifth day of gestation, diabetes was induced at the fasting state by a single intraperitoneal injection of streptozotocin (STZ; Sigma, St. Louis, MO, USA) in $0.1 \mathrm{mmol} / \mathrm{L}$ citrate buffer ( $\mathrm{pH} 4.5$ ) at a dose of $30 \mathrm{mg} / \mathrm{kg}$ bodyweight. Healthy pregnant rats received an equal volume of citrate buffer alone. Blood glucose was measured on D12 and D17 of pregnancy via tail by One Touch Glucometer (Johnson \& Johnson, USA). Diabetic pregnant rats, whose blood glucose ranged between 15 and $19 \mathrm{mmol} / \mathrm{L}$ both in two occasions, were included. Pregnant rats were housed individually and allowed to deliver spontaneously. Each litter size was reduced to six pups (three male and three female) to assure uniformity. The pups were weaned at three weeks of age. Respective 10 pups from healthy and GDM maternal rats were randomly selected to be sacrificed after weaning. The remaining offspring from healthy rats were fed an AIN-93 diet (7\% soybean oil) to 11 months old as a control offspring group (Con group). Offspring from GDM rats were randomly divided into three groups: GDM offspring (GDM group, fed 7\% soybean oil diet), GDM 
offspring with adequate $n-3$ PUFA diet (n-3 Adq-GDM group), and GDM offspring with deficient n-3 PUFA diet (n-3 Def-GDM group or high n-6 PUFA group). These rats were fed their respective diet until they were 11 months old. In the n-3 Adq-GDM group, 4\% of the 7\% of the soybean oil in the AIN-93 diet was replaced with fish oil containing 60\% n-3 PUFA (50\% docosahexaenoic acid (DHA), $10 \%$ eicosapentaenoic acid (EPA)) and the ratio of $n$ - 3 PUFA to $n-6$ PUFA was increased from 1:8 to 1.5:1. In then-3 Def-GDM group, $7 \%$ soybean oil was completely substituted by safflower oil, rendering an obvious decreased ratio of n-3/n-6 PUFA to 1:95. All purified diets were produced once a week (Medicience Ltd., Jiangsu, China) and kept at $-40{ }^{\circ} \mathrm{C}$. Diets were replaced every day and uneaten food was discarded. All rats were maintained with a $12 \mathrm{~h}$ light/dark cycle at $22{ }^{\circ} \mathrm{C}$ and had free access to food and water. Body weight and food intake were measured weekly. After overnight fasting, the animals were sacrificed at 11 months of age by intraperitoneal administration of chloral hydrate (350 mg/kg bodyweight). Blood was collected from the abdominal aorta and was centrifuged at $3000 \mathrm{r} / \mathrm{min}$ for $10 \mathrm{~min}, 4^{\circ} \mathrm{C}$. The livers were dissected and immediately placed into liquid nitrogen. They were subsequently stored at $-80{ }^{\circ} \mathrm{C}$ for analyses.

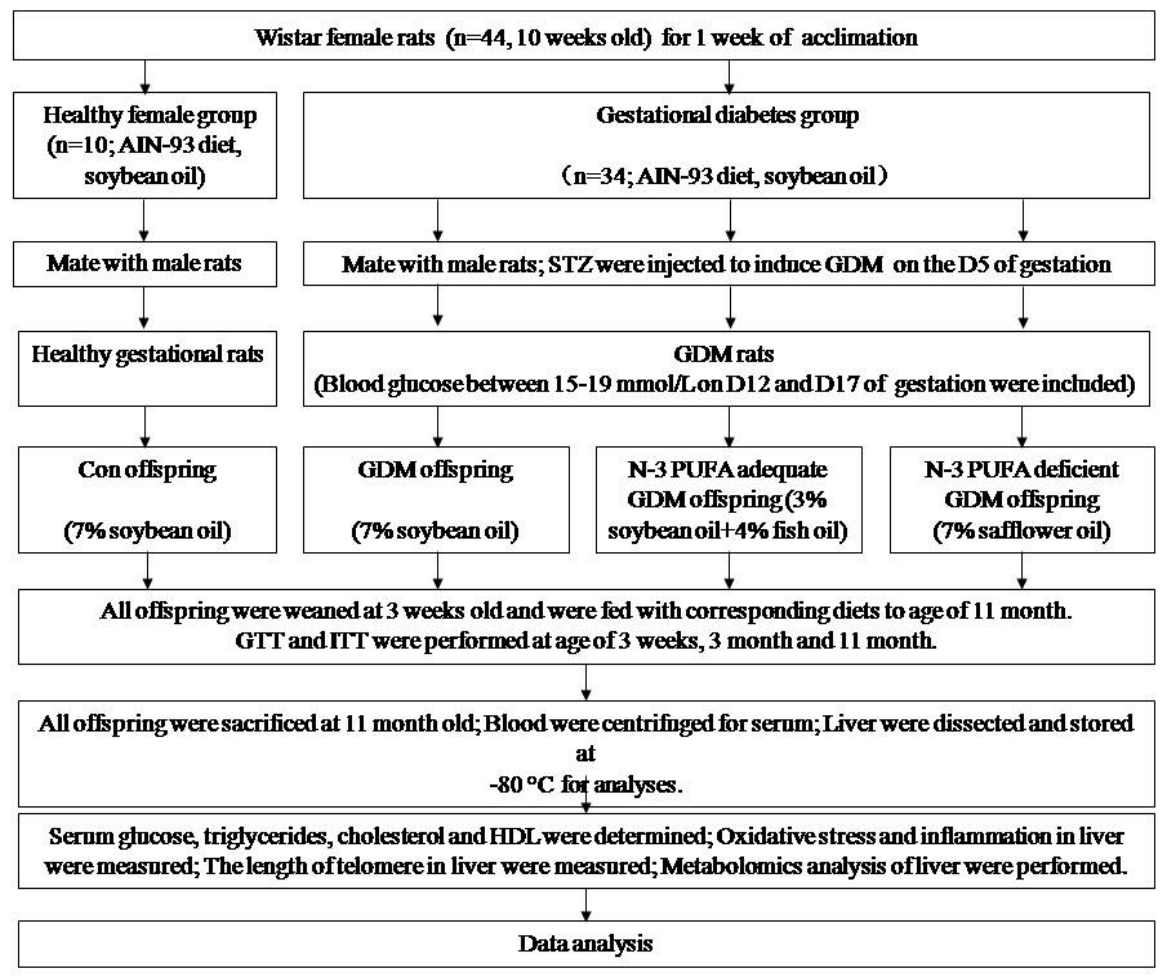

Figure 1. Flow diagram showing the protocol of the study.

\subsection{Determination of Serum Factors}

The levels of serum glucose, triglycerides, total cholesterol, and high-density lipoprotein (HDL) were determined by biochemical analyzer (Roche, Basel, Switzerland). Insulin was determined in duplicate using ELISA assays (Nanjing Jiancheng Bioengineering Institute, Nanjing, China) according to manufacturer's instructions.

\subsection{Glucose and Insulin Tolerance Tests}

Offspring glucose tolerance test (GTT) and insulin tolerance test (ITT) were performed at 3 weeks, 3 months, and 11 months (a week before being sacrificed). For GTT, glucose ( $2 \mathrm{~g} / \mathrm{kg}$ ) was intraperitoneally injected after $12 \mathrm{~h}$ overnight fasting. Blood glucose was measured via tail by glucometer at $0,15,30$, 60, and $120 \mathrm{~min}$. For ITT, insulin ( $0.5 \mathrm{unit} / \mathrm{kg})$ was intraperitoneally injected after $4 \mathrm{~h}$ fasting. Blood glucose was measured at $0,30,60,90$, and $120 \mathrm{~min}$. 


\subsection{Measurement of Oxidative Stress}

Liver tissues were homogenized using a multifunctional homogenizer. The protein concentrations were measured by colorimetric assay. The activity of superoxide dismutase (SOD), glutathione peroxidase (GSH-Px), and catalase (CAT) as well as the levels of malondialdehyde (MDA) and glutathione (GSH) were determined using commercial kits following manufacturer's instructions (Nanjing Jiancheng Biotechnology Institute, Nanjing, China) [21].

\subsection{Enzyme-Linked Immunosorbent Assay for Inflammatory Factors}

Commercially available ELISA kits (Nanjing Jiancheng Biotechnology Institute, Nanjing, China) were used to determine TNF- $\alpha$, IL-1 $\beta$, IL-6, and IL-10 levels in liver tissue of offspring rats in accordance with the manufacturer's instruction [22].

\subsection{Telomere Length Measurement}

DNA from the liver of offspring rats was isolated using DNA extraction kit (Tiangen, Beijing, China). Relative telomere lengths were measured by qPCR method with modifications [23]. In brief, DNA samples were used for telomeres and single-copy gene (36B4) qPCR, which was amplified by Power SYBR Green in $20 \mu \mathrm{L}$ total volume. The reaction was performed on a Light Cycler $480 \mathrm{II}$ with the following conditons: $95{ }^{\circ} \mathrm{C}$ for $3 \mathrm{~min}$, followed by 45 cycles of $95{ }^{\circ} \mathrm{C}$ for $3 \mathrm{~s}$ and $60{ }^{\circ} \mathrm{C}$ for $30 \mathrm{~s}$, followed by a dissociation stage to monitor amplification specificity. The telomere length was calculated based on $2^{-\Delta \Delta C t}$. The tested genes and primer sequence were $\left(5^{\prime} 3^{\prime}\right)$ : Tel1: GGTTTTTGAGGGTGAGGGTGAGGGTGAGGGTGAGGGT; Tel2: TCCCGACTATCCCTATCCCTATCCCTATCCCTATCCCTA; 36B4 Fw: CAGCAAGTGGG AAGGTGTAATCC; 36B4 Rv: CCCATTCTATCATCAACGGGTACAA.

\subsection{Metabolomics Analysis}

\subsubsection{Sample Preparation}

Non-targeted metabolomics analysis on liver tissue of offspring rats was performed. Twenty micrograms of each liver sample were weighed out into $2 \mathrm{~mL}$ EP tubes and homogenized with $800 \mu \mathrm{L}$ of prechilled $80 \%$ methanol by an automatic tissue homogenizer (Prima, London, UK). Homogenization cycles were for $40 \mathrm{~s}$ followed by cooling on dry ice and further $40 \mathrm{~s}$ homogenization and cooling. The mixtures were stored at $-20{ }^{\circ} \mathrm{C}$ for $1 \mathrm{~h}$, and then centrifuged at $14,000 \mathrm{~g}$ for $15 \mathrm{~min}$ at $4{ }^{\circ} \mathrm{C}$. The collected supernatants were filtered by a 0.22 um filtering membrane and transferred to auto sample vials (Waters Corporation, Milford, MA, USA) for analysis.

\subsubsection{High Performance Lipid Chromatography Coupled with Quadrupole-Time of Flight Mass} Spectrometry (HPLC-QTOF-MS) Analysis

HPLC-QTOF-MS analysis was performed on Waters XEVO G2 Q-TOF mass spectrometer system (Waters Corporation, USA). Separation was performed at $50{ }^{\circ} \mathrm{C}$ and $0.3 \mathrm{~mL} / \mathrm{min}$ using an Acquity UPLC BEH C18 (Waters Corporation, USA) column $(2.1 \times 100 \mathrm{~mm}, 1.7 \mathrm{um})$. The injection volume was $2 \mu \mathrm{L}$ and sample temperature was $4{ }^{\circ} \mathrm{C}$. A mixture of $0.1 \%$ formic acid in distilled water (mobile phase A) and $0.1 \%$ formic acid in acetonitrile (mobile phase B) was used. The initial eluent was $20 \% \mathrm{~B}$ for 0-3.0 min, followed by a linear gradient from $20 \%$ to $50 \%$ B for $7.0 \mathrm{~min}$. Mobile phase B was then increased from $50 \%$ to $90 \%$ over $7 \mathrm{~min}$ and subsequently to $100 \%$ for $3.0 \mathrm{~min}$. Mobile phase B was subsequently returned to initial conditions to re-equilibrate for $4.0 \mathrm{~min}$. MS acquisition was performed in positive ionization mode with a mass-to-charge $(\mathrm{m} / \mathrm{z})$ of $50-1200$. Source conditions were as follows: source temperature, $120^{\circ} \mathrm{C}$; desolvation temperature, $300{ }^{\circ} \mathrm{C}$; cone gas flow, $50 \mathrm{~L} / \mathrm{h}$; desolvation gas flow, $800 \mathrm{~L} / \mathrm{h}$. 


\subsubsection{Data Processing and Metabolites Identification}

LC/MC raw data were processed by the software Progenesis QI (Waters Corporation, USA) using the following parameters, retention time (RT) range $0.5-20 \mathrm{~min}$, mass range $50-1000 \mathrm{Da}$, and mass tolerance $0.01 \mathrm{Da}$. Isotopic peaks were excluded for analysis. The noise elimination level was at 10.00 , the minimum intensity was set to $15 \%$ of base peak intensity, and RT tolerance was at $0.01 \mathrm{~min}$. Metabolites identification was performed in Progenesis QI based on appropriate standards and mass fragmentation (MS/MS analysis). The available online database (HMDB) was used to identify the name and chemical structure of metabolites by molecular formula information. The file was obtained with three-dimensional data sets, including peak RT, peak intensities, and $\mathrm{m} / \mathrm{z}$. The resulting matrix was further reduced through removing peaks with missing value in more than $60 \%$ samples.

\subsection{Statistical Analysis}

Data are expressed as the mean \pm standard deviation (SD). Student's $t$-tests were used to determine significant differences between two groups. Significant differences between four groups were determined by a one-way ANOVA followed by an LSD analysis using SPSS 20.0. Differences were considered statistically significant if the $p$-value was less than 0.05 . For statistical analysis of data from metabolomics, the data that were pre-processed by the software Progenesis QI were transferred into SIMCA-P+14.0 (Umetircs, Umea, Sweden) to conduct multivariate statistical analysis. Principle component analysis (PCA) and orthogonal partial least-squares-discriminant analysis (OPLS-DA) were carried out to visualize the metabolic alterations among groups. Metabolites with $p<0.05$ and variable importance on projection (VIP) $>1$ were considered relevant for group discrimination.

\section{Results}

\subsection{Serum Biochemical Index of GDM Offspring and Effect of n-3 PUFA}

As shown in Table 1, at weaning, there was no significant difference in fasting and postprandial glucose between Con and GDM offspring. The level of insulin of GDM offspring at weaning was significantly decreased in the fasting state $(p<0.05)$, compared to that of Con offspring. However, in the postprandial state, insulin of GDM offspring was significantly increased $(p<0.01)$, indicating early dysfunction of $\beta$ cells caused by severe maternal hyperglycemia. No significant changes were observed in triglyceride and total cholesterol between the Con offspring and GDM offspring at weaning. As shown in Table 2, when GDM offspring grew to an age of 11 months, the fasting insulin level was still significantly decreased $(p<0.01)$ with a higher fasting glucose level $(p>0.05$, not significant) compared to that of Con offspring. N-3 Adq-GDM offspring exhibited significantly increased insulin $(p<0.01)$ compared to that of GDM offspring. No improvement of insulin was observed in n-3 Def-GDM group. Supplementation of $n$-3 PUFA significantly decreased triglyceride and total cholesterol in serum of GDM offspring $(p<0.05$ and $p<0.01)$. Meanwhile, $n-3$ PUFA also significantly decreased the ratio of total cholesterol to high-density lipoprotein (TC/HDL), which was a more meaningful result than the value of HDL alone.

Table 1. Biochemical index of gestational diabetes mellitus (GDM) offspring at weaning.

\begin{tabular}{ccc}
\hline 3 Week (Weaning) & Con & GDM \\
\hline Fasting glucose $(\mathrm{mmol} / \mathrm{L})$ & $6.17 \pm 1.24$ & $6.01 \pm 1.3$ \\
Postprandial glucose (mmol/L) & $7.34 \pm 0.61$ & $7.57 \pm 0.49$ \\
Fasting insulin $(\mathrm{mU} / \mathrm{L})$ & $12.01 \pm 0.59$ & $9.7 \pm 0.46^{*}$ \\
Postprandial insulin $(\mathrm{mU} / \mathrm{L})$ & $13.23 \pm 0.78$ & $20.17 \pm 1.45^{* *}$ \\
TG $(\mathrm{mmol} / \mathrm{L})$ & $0.45 \pm 0.1$ & $0.46 \pm 0.11$ \\
TC $(\mathrm{mmol} / \mathrm{L})$ & $1.76 \pm 0.19$ & $1.98 \pm 0.16$
\end{tabular}

Data are presented as mean \pm SD. $n=8-10$ offspring rats/group. ${ }^{*} p<0.05,{ }^{* *} p<0.01$ vs. Control offspring (Con). 
Table 2. Biochemical index of GDM offspring at 11 months of age and n-3 polyunsaturated fatty acids (n-3 PUFA) effect.

\begin{tabular}{ccccc}
\hline 11 Months old & Con & GDM & n-3 Adq-GDM & n-3 Def-GDM \\
\hline Glucose (mmol/L) & $6.05 \pm 0.37$ & $8.39 \pm 2.07$ & $6.3 \pm 0.54$ & $8.38 \pm 1.66$ \\
Insulin (mU/L) & $11.73 \pm 0.55$ & $8.03 \pm 0.47^{* *}$ & $10 \pm 0.46^{* \# \#}$ & $7.8 \pm 0.78^{* *}$ \\
TG (mmol/L) & $0.79 \pm 0.13$ & $0.63 \pm 0.19$ & $0.43 \pm 0.19^{* \#}$ & $0.61 \pm 0.19$ \\
TC (mmol/L) & $1.87 \pm 0.04$ & $1.79 \pm 0.36$ & $0.69 \pm 0.16^{* * \# \#}$ & $1.7 \pm 0.17$ \\
HDL (mmol/L) & $0.53 \pm 0.03$ & $0.52 \pm 0.18$ & $0.27 \pm 0.04^{* * \# \#}$ & $0.47 \pm 0.03$ \\
TC/HDL & $3.52 \pm 0.12$ & $3.44 \pm 0.66$ & $2.55 \pm 0.28^{* * \# \#}$ & $3.62 \pm 0.19$ \\
\hline
\end{tabular}

Data are presented as mean \pm SD. $n=8-10$ offspring rats/group. ${ }^{*} p<0.05,{ }^{* *} p<0.01$, vs. Control offspring (Con); ${ }^{\#}$ $p<0.05,{ }^{\#} p<0.01$, vs. GDM offspring (GDM).

\subsection{Glucose and Insulin Tolerance Test during Growth of GDM Offspring and Effect of n-3 PUFA}

GTT and ITT were performed to evaluate the risk of developing diabetes during growth of GDM offspring. The diabetic risk in GDM offspring gradually increased with age. At weaning, no obvious GTT and ITT differences were observed (Figure 2A,D). At 3 months of age, GDM offspring exhibited a higher blood glucose level only at $15 \mathrm{~min}$ in GTT, compared to that of Con offspring (Figure 2B). In ITT, the blood glucose level of GDM offspring was higher only at $30 \mathrm{~min}$ and $60 \mathrm{~min}$ (Figure 2E). Surprisingly, when GDM offspring grew to 11 months of age, the GTT was obviously impaired. Furthermore, GDM offspring receiving ann-3 PUFA deficient diet exhibited an even more obvious impairment in GTT. GDM offspring with adequate $n$-3 PUFA diet; however, showed a significant decrease in the GTT (Figure 2C). Likewise, $n$-3 PUFA supplementation also decreased the ITT in GDM offspring at 11 months of age, whereas the worst ITT was observed in $n$-3 PUFA deficient offspring (Figure 2F).

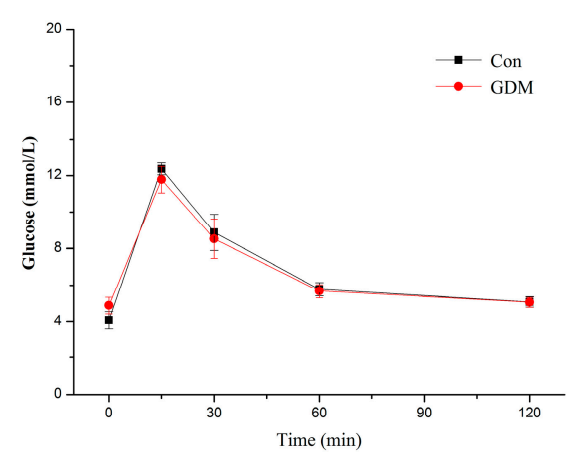

(A)

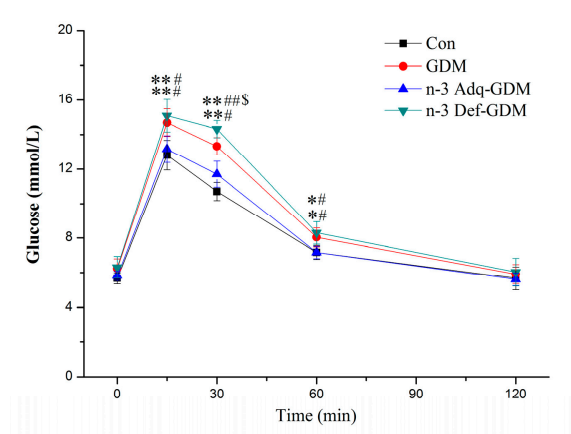

(C)

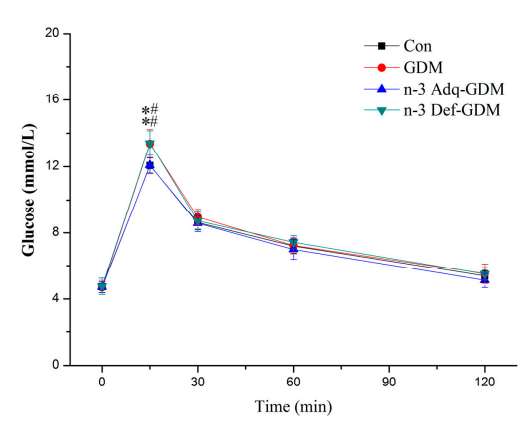

(B)

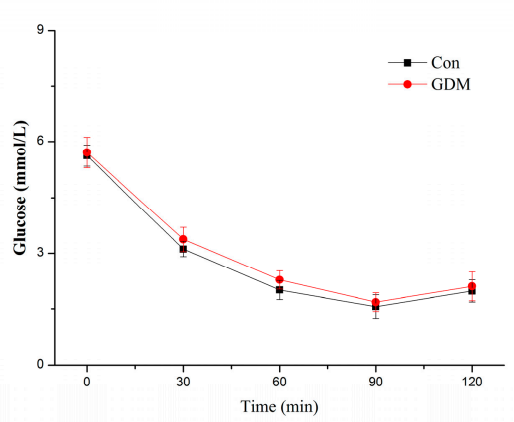

(D)

Figure 2. Cont. 


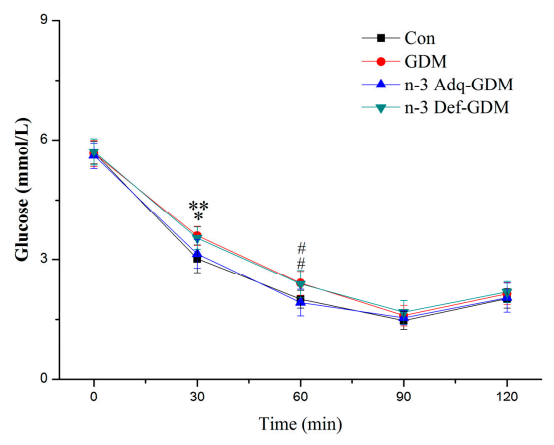

(E)

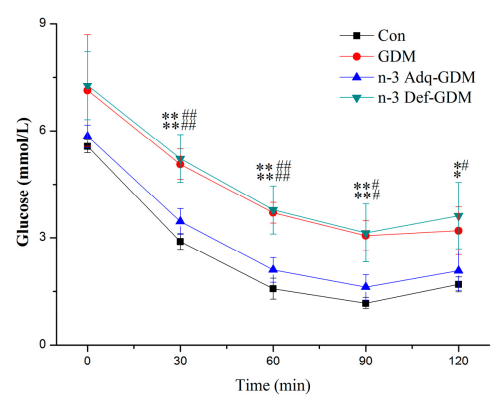

(F)

Figure 2. Glucose tolerance test (GTT) and insulin tolerance test (ITT) in growing gestational diabetes mellitus (GDM) offspring and the effect of n-3 polyunsaturated fatty acids ( $n-3$ PUFA). (A) GTT at weaning; (B) GTT at 3 months; (C) GTT at 11 months; (D) ITT at weaning; (E) ITT at 3 months; (F) ITT at 11 months. Values are the mean \pm SD. $n=8$ rats/group. ${ }^{*} p<0.05,{ }^{* *} p<0.01$, vs. Control offspring (Con); ${ }^{\#} p<0.05,{ }^{\# \#} p<0.01$, vs. n-3 Adequate-GDM offspring (n-3 Adq-GDM); ${ }^{\$} p<0.05, \mathrm{n}-3$ Deficient-GDM offspring (n-3 Def-GDM) vs. GDM offspring (GDM).

\subsection{Effect of n-3 PUFA on Oxidative Stress of the Liver of GDM Offspring}

Oxidative stress in the liver was measured (Figure 3). The glutathione (GSH) level, the activity of superoxide dismutase (SOD) and glutathione peroxidase (GSH-Px) were decreased in the liver of GDM offspring ( $p<0.01, p<0.01$ and $p<0.05$, respectively). $n-3$ PUFA significantly enhanced the activity of SOD and catalase (CAT) in the liver of GDM offspring $(p<0.01)$. No improvement was observed in n-3 Def-GDM offspring and there was a differential effect on activity of SOD and GSH-Px between n-3 Adq-GDM offspring and n-3 Def-GDM offspring ( $p<0.01$ and $p<0.05$ ).

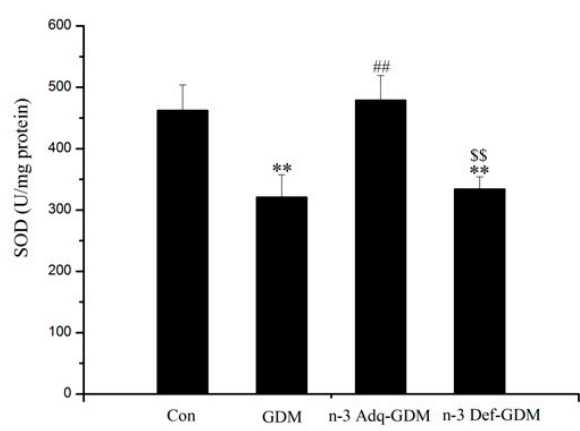

(A)

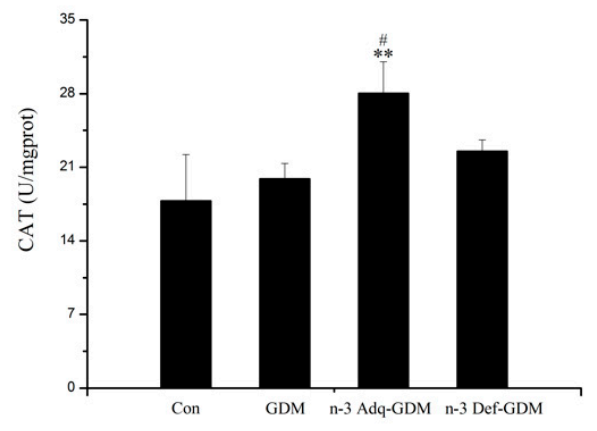

(C)

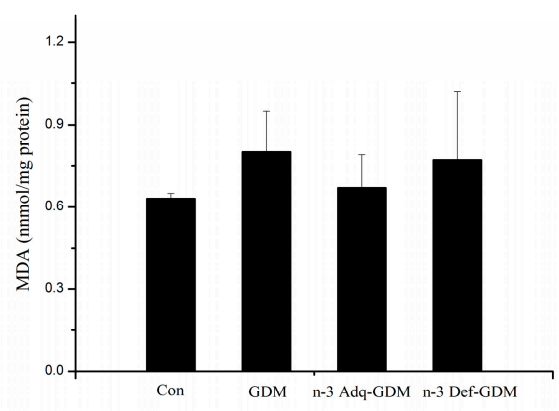

(B)

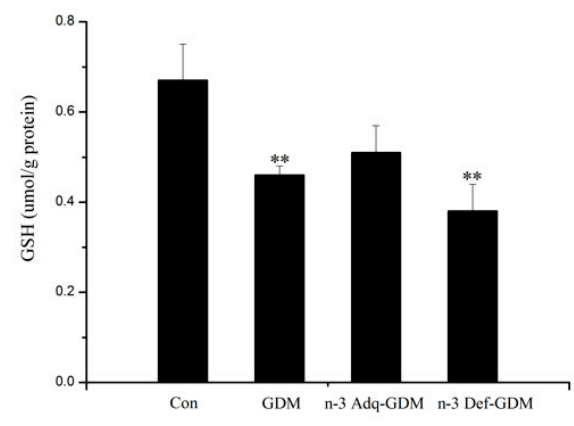

(D)

Figure 3. Cont. 


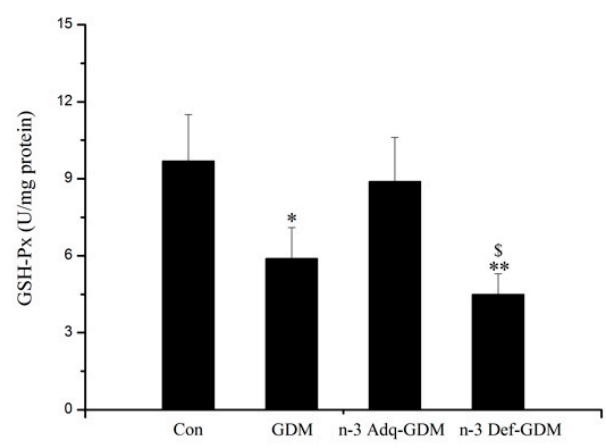

(E)

Figure 3. Oxidative stress in the liver of gestational diabetes mellitus (GDM) offspring at 11 months of age and the effect of $n-3$ polyunsaturated fatty acids ( $n-3$ PUFA) on oxidative stress. (A)The activity of SOD, (B) MDA, (C) The activity of CAT, (D) GSH, (E) The activity of GSH-Px. Bars are mean \pm SD. $n=8$ rats/group. ${ }^{*} p<0.05,{ }^{* *} p<0.01$, vs. Control offspring (Con); ${ }^{\#} p<0.05,{ }^{\# \#} p<0.01$, vs. GDM offspring (GDM); ${ }^{\$} p<0.05, \$ \$ p<0.01$, vs. n-3 Adequate-GDM offspring (n-3 Adq-GDM).

\subsection{Effect of n-3 PUFA on Inflammatory Factors in the Liver of GDM Offspring}

As shown in Figure 4, the levels of hepatic TNF- $\alpha$ and IL-1 $\beta$ were increased in GDM offspring $(p<0.05)$. The level of IL-10, an anti-inflammatory cytokine, was decreased in the liver of GDM offspring $(p<0.01)$. $n-3$ PUFA decreased IL-1 $\beta$ and increased IL-10 levels $(p<0.05)$. N-3 Def-GDM offspring exhibited the highest level of TNF- $\alpha$ and IL- $1 \beta$ in all groups. There was a significant opposite trend in IL-1 $\beta(p<0.01)$ and IL-10 $(p<0.01)$ between the n-3 Adq-GDM group and n-3 Def-GDM group. No significant difference was observed in IL-6 levels.

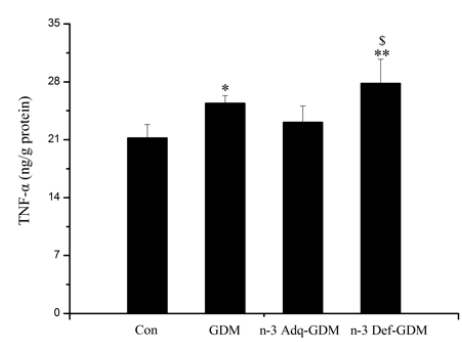

(A)

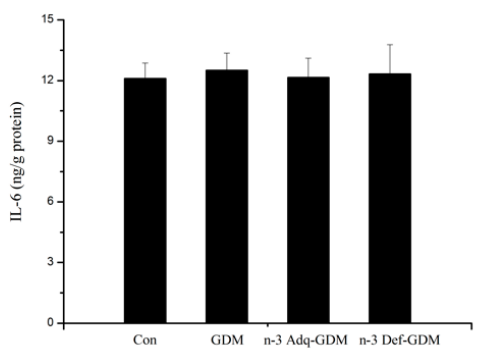

(C)

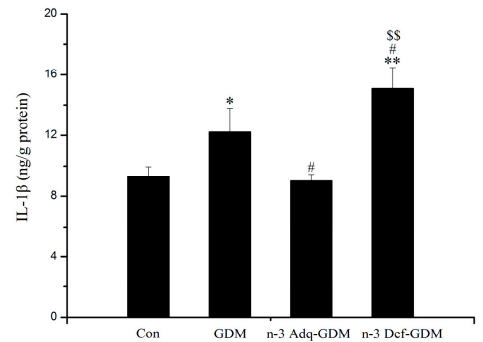

(B)

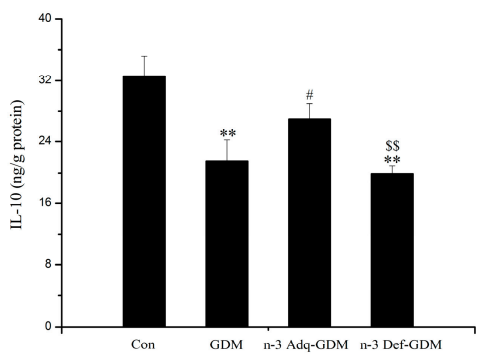

(D)

Figure 4. Inflammatory biomarkers in the liver of gestational diabetes mellitus (GDM) offspring at 11 months of age and the effect of $\mathrm{n}-3$ polyunsaturated fatty acids ( $n$-3 PUFA) on inflammatory biomarkers. (A) TNF- $\alpha$, (B) IL-1 $\beta$, (C) IL-6, (D) IL-10. Bars are mean \pm SD. $n=8$ rats/group. ${ }^{*} p<0.05,{ }^{* *} p<0.01$, vs. Control offspring (Con); ${ }^{*} p<0.05$, vs. GDM offspring (GDM); ${ }^{\$} p<0.05, \$ \$ p<0.01$, vs. n-3 Adequate-GDM offspring (n-3 Adq-GDM). 
3.5. Influence of GDM on Telomere Length of the Liver of Offspring and the Effect of n-3 PUFA and n-6 PUFA on Telomere Length

At weaning, there was no significant difference in length of the hepatic telomeres between Con offspring and GDM offspring (Figure 5A). However, as shown in Figure 5B, telomere length of the liver of GDM offspring at 11 months of age was nearly significantly decreased, compared with that of Con group ( $p=0.058$ ). Furthermore, telomere length of the liver of $n-3$ Def-GDM offspring was significantly shortened, compared with that of Con offspring $(p<0.05)$. Additionally, n-3 Def-GDM offspring exhibited the shortest hepatic telomere length of all four groups. The telomere length of the liver of n-3 Adq-GDM offspring was nearly improved by supplementation with $n-3$ PUFA, compared with that of GDM offspring (not significant, nearly significant, $p=0.081$ ). It is noticeable that there is an obvious opposite trend of hepatic telomere length between n-3 Adq-GDM offspring and n-3 Def-GDM offspring $(p<0.05)$, suggesting a differential effect of $n-3$ PUFA and $n-6$ PUFA on the telomere length of the liver of GDM offspring.

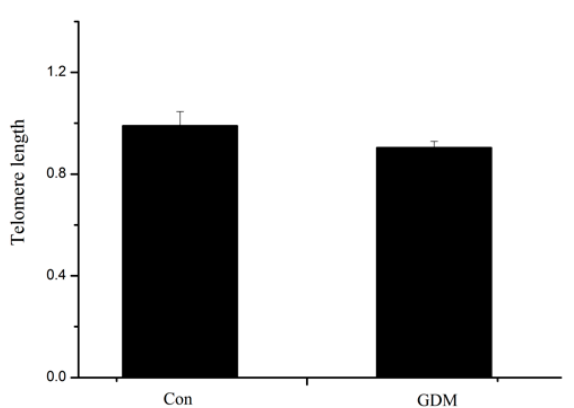

(A)

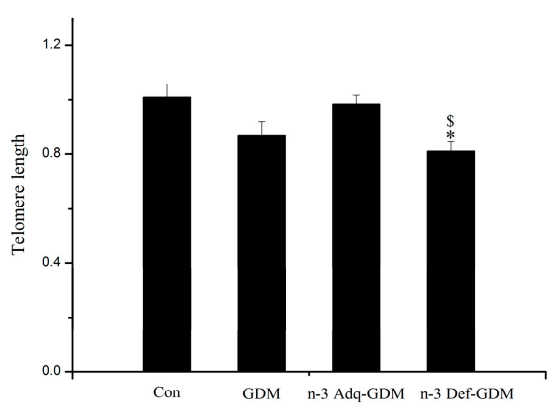

(B)

Figure 5. Influence of gestational diabetes mellitus (GDM) on telomere length (TL) of the liver of offspring and the effect of $\mathrm{n}-3$ polyunsaturated fatty acids ( $n-3$ PUFA) and $n-6$ PUFA on telomere length of the liver of GDM offspring. (A) At weaning; (B) at 11 months. $n=8$ rats/group. ${ }^{*} p<0.05$, vs. Control offspring (Con); $\$ p<0.05$, vs. n-3 Adequate-GDM offspring (n-3 Adq-GDM).

3.6. Metabolomics Analysis of the Liver of GDM Offspring at Old Age and the Modulating Effect of n-3 PUFA on the Liver of GDM Offspring

Samples of the liver were clustered within their respective groups in a PCA model and further clustered in OPLS-DA model (Supplementary Figure S1). Pairwise OPLS-DA modeling was conducted to assess data quality and to screen differential metabolites. The value of $R^{2} Y$ and $Q^{2} Y$ were high, indicating a good model quality (Supplementary Table S1). A permutation test was performed to further confirm the model's validity (Supplementary Figure S1). For maximum screening of all significantly altered metabolites, metabolites were selected based only on variable importance in projection (VIP) values of greater than 1 and $p<0.05$, without further screening by fold change.

Metabolomics analysis revealed obvious metabolic alterations of the liver when GDM offspring grew to 11 months of age. Some representative metabolites were closely related to oxidative stress, inflammation and risk of developing diabetes (Table 3). A total of 73 metabolites were significantly altered in the liver of GDM offspring, with 38 increased metabolites and 35 decreased ones (Supplementary Table S2 and Table 3). Twenty-seven metabolites of the altered 73 metabolites were improved in the n-3 Adq-GDM group. Only seven metabolites in the liver of n-3 Def-GDM group were improved but changing trend of other 21 metabolites were aggravated. Obviously, more metabolites were improved in the n-3 Adq-GDM group rather than in the high n-6 PUFA group (n-3 Def-GDM group). $n-3$ PUFA played an important role in modulating metabolism of the liver of GDM offspring. 
Table 3. Representative metabolites altered in the liver of gestational diabetes mellitus (GDM) offspring at 11 months of age and the effects of n-3 polyunsaturated fatty acids (n-3 PUFA) and $n-6$ PUFA on them.

\begin{tabular}{|c|c|c|c|c|c|c|}
\hline \multirow[b]{2}{*}{ Identification } & \multirow[b]{2}{*}{ RT (Min) } & \multirow[b]{2}{*}{$\mathrm{m} / \mathrm{z}$} & \multicolumn{3}{|c|}{ Changing Trend } & \multirow[b]{2}{*}{ Significance } \\
\hline & & & $\begin{array}{l}\text { GDM vs. } \\
\text { Con }\end{array}$ & $\begin{array}{l}\text { n-3adq vs. } \\
\text { GDM }\end{array}$ & $\begin{array}{l}\text { n-3def vs. } \\
\text { GDM }\end{array}$ & \\
\hline $\begin{array}{l}\text { Ceramide } \\
\text { (d18:1/16:0) }\end{array}$ & 17.78 & 560.5073 & $\uparrow$ & $\downarrow^{*}$ & - & $\begin{array}{l}\text { Biomarker for diabetes; impair } \\
\text { insulin signaling and cause } \\
\text { insulin resistance; increase } \\
\text { oxidative stress; promote } \\
\text { inflammation; contribute to } \\
\text { non-alcohol fatty liver disease }\end{array}$ \\
\hline $\begin{array}{l}\text { Tetrahydro-11 } \\
\text {-deoxycortisol }\end{array}$ & 11.56 & 337.2756 & $\uparrow$ & $\downarrow^{* *}$ & - & $\begin{array}{l}\text { Impact cortisol and further } \\
\text { impact insulin production and } \\
\text { glucose metabolism; inhibit } \\
\text { glycogen synthesis; cause } \\
\text { insulin resistance }\end{array}$ \\
\hline $\begin{array}{l}\text { 9'-Carboxy- } \gamma \\
\text {-tocotrienol }\end{array}$ & 16.50 & 395.2234 & $\downarrow$ & - & $\downarrow^{* *}$ & $\begin{array}{l}\text { Antioxidant effect; improve } \\
\text { glycemic control; prevent } \\
\text { hyperlipidemia; suppress } \\
\text { inflammation }\end{array}$ \\
\hline$\alpha$-Linolenic acid & 11.93 & 570.3029 & $\downarrow$ & - & $\downarrow^{* *}$ & $\begin{array}{c}\text { Decreases diabetic risk; } \\
\text { improve insulin resistance; } \\
\text { improve oxidative stress and } \\
\text { inflammation; regulate lipid } \\
\text { metabolism; improve } \\
\text { non-alcohol fatty liver disease }\end{array}$ \\
\hline $\begin{array}{l}\text { Hexadecenoic } \\
\text { acid }\end{array}$ & 15.99 & 271.2649 & $\uparrow$ & - & - & $\begin{array}{l}\text { Induce endoplasmic reticulum } \\
\text { stress and insulin resistance; } \\
\text { lipotoxicity; enhance oxidative } \\
\text { stress and inflammation; } \\
\text { contribute to non-alcohol fatty } \\
\text { liver disease } \\
\end{array}$ \\
\hline Niacinamide & 0.95 & 123.0515 & $\downarrow$ & - & - & $\begin{array}{c}\text { Prevent diabetes; protect } \beta \text { cell; } \\
\text { antioxidative role; } \\
\text { anti-inflammatory effect }\end{array}$ \\
\hline Oxalacetic acid & 18.17 & 154.9962 & $\downarrow$ & - & $\downarrow^{*}$ & $\begin{array}{l}\text { Impact citric acid cycle and } \\
\text { glucose and lipid metabolism; } \\
\text { decrease of it indicate } \\
\text { gluconeogenesis }\end{array}$ \\
\hline Phenylethylamine & 2.52 & 122.0956 & $\uparrow$ & - & $\uparrow^{* *}$ & $\begin{array}{l}\text { Indicate possibility of hepatic } \\
\text { damage and hepatic } \\
\text { encephalopathy }\end{array}$ \\
\hline
\end{tabular}

The " $\uparrow$ " and " $\downarrow$ " arrows represent a significant increasing or decreasing trend of metabolites of GDM offspring. Green arrows show modulating effect on altered metabolites, and the red shows aggravating results. "_-" means no significant change. ${ }^{*} p<0.05,{ }^{* *} p<0.01$, vs. GDM offspring.

Meanwhile, the enrichment analysis of multiple metabolic pathways was performed (Supplementary Figure S2). Some of these altered metabolic pathways were closely related to glucose and lipid metabolism, and diabetes risk, including citric acid cycle, pyruvate metabolism, glycolysis, gluconeogenesis, linoleic acid, and linolenic acid metabolism.

\section{Discussion}

Long-term effects of GDM on offspring are poorly understood. We studied the risk of developing diabetes during growth of GDM offspring, particularly as they grew to an old age. To study the long-term diabetic risk of GDM offspring, we evaluated two hypotheses. The first is that GDM can lead to aging of the liver of offspring, which increases the risk of developing diabetes. To evaluate this hypothesis, we measured oxidative stress, inflammation, and telomere length. The second is that GDM can cause long-term metabolic changes in the liver of offspring. If so, we evaluate whether such changes are associated with diabetic risk. The discussion is primarily based on these two hypotheses (Figure 6). Meanwhile, the effect of $n-3$ PUFA on the diabetic risk in GDM offspring was also studied. 
Oil replacement is a common method to study the effect of $n-3$ PUFA. According to data determined in our laboratory, the ratio of n-3 PUFA to n-6 PUFA in diets of the Con group, n-3 Adq-GDM group, and n-3 Def-GDM group (high n-6 PUFA group) were 1:8, 1.5:1, and 1:95, respectively. Metabolomics performed by UPLC-QTOF-MS detected changes of DHA in the liver. The mean abundance value of DHA in the Con, GDM, n-3 Adq-GDM, and n-3 Def-GDM groups were 3429, 4422, 15966, and 1356, respectively (Con vs. GDM, not significant; n-3 Adq-GDM group vs. Con, GDM and n-3 Def group, $p<0.01 ; n-3$ Def-GDM group vs. Con, GDM and n-3 Adq-GDM group, $p<0.01$ ), showing that fed n-3 fatty acids from fish oil diet were successfully incorporated into n-3 Adq-GDM offspring.

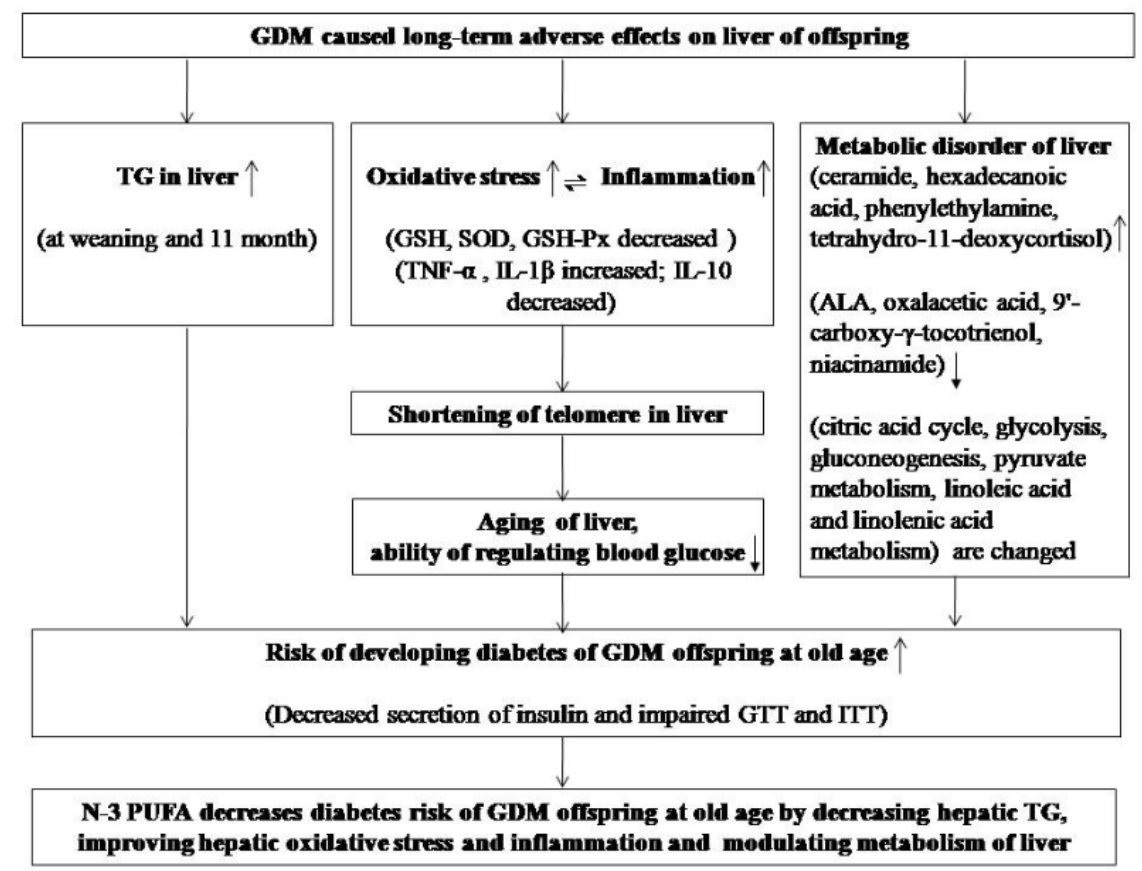

Figure 6. A summary figure showing all of the results.

Low birth weight is associated with adult diseases including type 2 diabetes [24]. In the present study, the birth weight of GDM offspring was significantly decreased (Supplementary Figure S3), suggesting their increased risk of developing diabetes later in life. GDM offspring exhibited a lifelong growth restriction, which was improved in the n-3 Adq-GDM group, but not in the n-3 Def-GDM group, indicating a protective effect of $n-3$ PUFA on GDM offspring. Moreover, triglyceride levels were increased in the liver of GDM offspring at weaning and even progressed up to 11 months of age (Supplementary Figure S4). Hepatic triglycerides have been associated with insulin resistance and diabetes risk [25]. Troy Pereira found increased TG in the liver of "obese" offspring from "obese" GDM rats [7]. However, we found that hepatic TG was increased in "lean" offspring from "lean" GDM rats induced by STZ. This result indicates that regardless of the GDM model, intrauterine hyperglycemia can lead to hepatic lipid metabolism disorders in offspring, and these disorders can even continue to old age, which increases the risk of developing diabetes. $n$ - 3 PUFA decreased the levels of triglyceride and cholesterol in the liver of GDM offspring, suggesting a protective effect on diabetic risk in GDM offspring.

Studies have shown that the risk of developing diabetes in GDM offspring was different. A ref et al. found abnormal fasting glucose in offspring rats at postnatal weeks one and two [26]. In Ding's study, GTT was impaired in 8-weeks-old offspring mice [27]. Kamel et al. found impaired GTT in offspring rats at 15 weeks of age [28]. Population studies found that the diabetic risk in GDM offspring was increased at childhood, puberty, and adulthood [29-31]. However, longer-term diabetic risk, even after the offspring have grown to an old age, has been poorly studied. Furthermore, the relationship between n-3 PUFA and diabetes risk varies from study to study, even with opposite conclusions [3,4]. 
Our results showed that the diabetes risk of GDM offspring gradually increased, with no obvious change in the GTT and ITT at weaning to slight changes at 3 months old. Surprisingly, GDM offspring exhibited obvious impairment in the GTT and ITT at 11 months. $n-3$ PUFA improved GTT and ITT of GDM offspring at 11 months, whereas more obviously impaired GTT and ITT were observed in n-3 Def-GDM offspring, indicating the beneficial effect of $n-3$ PUFA on the diabetic risk in GDM offspring.

Our present study showed that the liver of GDM offspring exhibited oxidative stress and inflammation at 11 months of age, which were important factors in inducing diabetic risk. SOD is an important defense enzyme catalyzing dismutation of superoxide radicals [32]. The decreased activity of SOD in the liver of GDM offspring lowers cellular capacity to scavenge free radicals, which reduces hepatic cell protection from oxidant exposure, resulting in diabetic risk. In the diabetic state, glucose is preferentially utilized in the polyol pathway, which consumes nicotinamide adenine dinucleotide phosphate (NADPH) necessary for GSH regeneration. Thus, there is a link between diabetes and GSH depletion [32]. Furthermore, low levels of GSH could directly decrease activity of GSH-Px, which is a main antioxidant enzyme that protects cells against hydroperoxides caused by reactive ROS [33]. Therefore, decreased GSH levels and GSH-Px activity in the liver of GDM offspring were factors for developing diabetes. $n-3$ PUFA increased the activity of SOD and it also increased the activity of CAT, which reduces intracellular ROS and inhibits oxidative damage [34], preserving its protective role in diabetes development. TNF- $\alpha$ is one of the most important pro-inflammatory mediators that is critically involved in the pathogenesis of diabetes by inducing tissue-specific inflammation via activation of various pathways such as NF- $\mathrm{kB}$. TNF- $\alpha$ decreases GLUT4 and phosphorylation of IRS-1 [35]. IL-1 $\beta$ is also a key contributor to the pathogenesis of diabetes. It contributes to insulin resistance in the liver [36]. TNF- $\alpha$ and IL-1 $\beta$ were increased in the liver of GDM offspring, whereas IL-10, an anti-inflammatory cytokine, was decreased in GDM offspring, indicating an increased risk of developing diabetes. There was a significantly opposite trend in TNF- $\alpha$, IL-1 $\beta$, and IL-10 between the n-3 Adq-GDM group and n-3 Def-GDM group, which could lead to differential diabetes risk.

Furthermore, oxidative stress and inflammation are important factors leading to telomere shortening $[37,38]$. The length of telomere in the liver of GDM offspring was nearly significantly shortened at 11 months of age $(p=0.058)$, indicating potential aging of the liver. A population study found that telomere length was shortened in leucocytes of the fetus from mothers with GDM, suggesting the possibility of aging of GDM offspring in the future [39]. However, other studies showed that no significant telomere shortening was observed in neonatal offspring and adult offspring from mothers with diabetes [40,41]. We observed a nearly shortened telomere length in the liver when GDM offspring grew to 11 months of age. It can be speculated that if the rats continued to age past 11 months, the length of telomeres in the liver of GDM offspring would be significantly shortened and hepatic aging may be more obviously. Because the liver is the main organ for the regulation of blood glucose, hepatic aging of GDM offspring may reduce the liver's ability to regulate blood glucose, resulting in risk of developing diabetes. We observed an opposite result on the length of the hepatic telomeres in the liver between n-3 Adq-GDM offspring and n-3 Def-GDM offspring, suggesting that $n-3$ PUFA and $n-6$ PUFA played a differential role in telomere length. GDM offspring with an-3 PUFA deficient diet exhibited the shortest telomere length, whereas $n-3$ PUFA postponed shortening of the telomere.

Our study is the first to investigate the long-term influence of GDM on metabolic changes in the liver of offspring. Surprisingly, many metabolites were altered in the liver when GDM offspring grew to 11 months. Some typical metabolites were closely related to diabetes risk. A particularly novel finding was that ceramide was increased in the liver of GDM offspring. Ceramide is a typical biomarker for diabetes risk. Excessive accumulation of ceramide in the liver impairs insulin signaling and contributes to diabetic development [42]. Moreover, hexadecenoic acid was increased in the liver of GDM offspring, which could induce lipotoxicity and insulin resistance, increasing the risk of developing type 2 diabetes [43]. It is worth noting that oxaloacetic acid was also decreased in the liver of GDM offspring, which directly affects the citric acid cycle and further affects glucose and lipid metabolism. A decrease inoxaloacetic acidalso might indicate abnormal gluconeogenesis [44]. 
Tetrahydro-11-deoxycortisol was increased in the liver of GDM offspring, which could further increase the level of cortisol. Cortisolcan affect insulin production and glucose metabolism and it can cause insulin resistance [45,46]. $\alpha$-linolenic acid (ALA) can improve tissue's insulin resistance [47,48]. ALA is also associated with the lowest risk of type 2 diabetes [49]. ALA was decreased in the liver of GDM offspring, which might be related to the risk of developing diabetes. Niacinamide can be used to prevent the development of diabetes by reducing $\beta$-cell apoptosis [50-52]. Niacinamide was decreased in the liver of GDM offspring, which might be a risk factor for the development of diabetes. Meanwhile, it is worth noting that some altered metabolites were not only related to diabetic risk, but also were associated with oxidative stress, inflammation, aging, and hepatic function, such as 9'-carboxy-gamma-tocotrienol [53,54], $\alpha$-linolenic acid, hexadecenoic acid [55], ceramide [56], niacinamide $[57,58]$, and phenylethylamine $[59,60]$. These metabolites were consistent with the results of the enhanced oxidative stress and inflammation, and shortening of telomeres in the liver of GDM offspring at an old age. Thus, the second hypothesis based on metabolomics was not isolated, but rather linked with the first hypothesis.

All metabolites altered in the liver of GDM offspring involve various metabolic pathways, some of which were closely related to glucose and lipid metabolism, as well as diabetic risk, such as citric acid cycle, pyruvate metabolism, glycolysis, gluconeogenesis, linoleic acid, and linolenic acid metabolism (Supplementary Figure S4). Our resultsindicate that hyperglycemia uterine conditions, which the fetus experienced early in life, did cause long-term metabolic disorders to the liver of offspring later in life. The liver is the center of metabolism and a key site for metabolic disorders in patients with type 2 diabetes. Compared with the possibility of results explained by transcriptome and proteomics, metabolomics can explain what ultimately happened. Thus, the changes of these metabolites and metabolic pathways demonstrated an important molecular basis and mechanism for the increased risk of GDM offspring at old age. Our results also provided evidence and a molecular basis for the theory of developmental origins of health and disease. $n$-3 PUFA played a role in improving the overall metabolism of the liver of GDM offspring. Changing trends of some metabolites were even aggravated in the high $n-6$ PUFA group. For the first time, the differential effects of $n-3$ PUFA and $n-6$ PUFA on hepatic metabolism of GDM offspring were compared in this study.

It is worth noting that the overall physical condition of GDM offspring was very poor. Limbs of GDM offspring were thin at weaning and 11 months old (Figure 7). Some of the n-3Def-GDM offspring even could not stand properly, which may be partly because of muscle weakness. This observed muscle weakness implies that these animals are unable to perform adequate exercise, which in turn may also enhance insulin resistance and predispose them to develop diabetes. It is possible that the muscle tissues of these animals have fewer number of mitochondria and that actin-myosin and filaments may be improperly organized. The fatty acid pattern of muscle tissue may be different. Whereas, the performance of n-3 Adq-GDM offspring was improved. This could be partly because $n-3$ PUFA improved insulin resistance in muscle tissue in GDM offspring, which deserves further investigation.

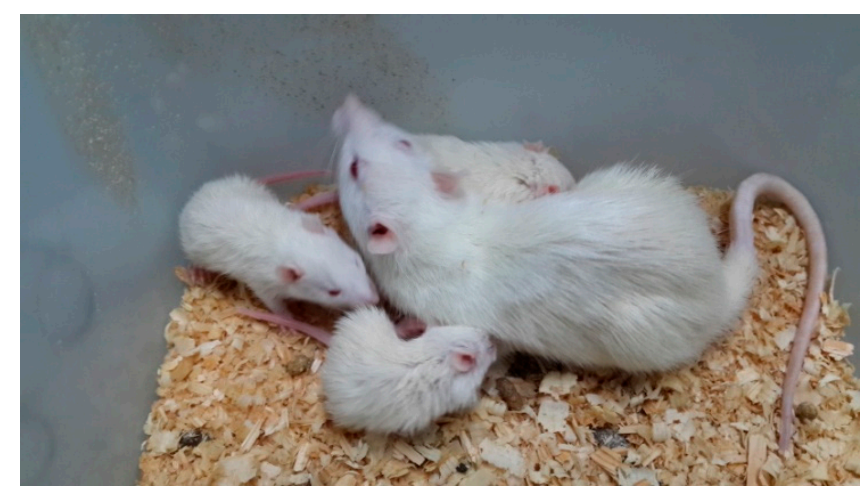

Figure 7. The gestational diabetes mellitus (GDM) offspring at weaning. 
In summary, GDM offspring exhibited an obvious increased risk for diabetes when they grew to an old age, whereas $n-3$ PUFA decreased this risk. To the best of our knowledge, this is the first study to investigate the long-term influence of GDM on metabolism of the liver of offspring and the modulating effect of $n$-3 PUFA on hepatic metabolism. Surprisingly, the differential effect of $n-3$ PUFA and $n-6$ PUFA on the length of hepatic telomeres in GDM offspring was discovered in this study. Finally, our results also provide evidence and a molecular basis for the $\mathrm{DOHaD}$ theory and fetal programming hypothesis.

Supplementary Materials: The following are available online at http:/www.mdpi.com/2072-6643/11/7/1699/s1. Figure S1. Effect of $n-3$ PUFA on body weight of gestational diabetes mellitus (GDM) offspring; Figure S2. Effect of $n-3$ PUFA on TG and TC in the liver of gestational diabetes mellitus (GDM) offspring; Figure S3. Score plots of PCA and OPLS-DA of all groups and pairwise OPLS-DA between groups and corresponding permutation tests of the liver when offspring grew to 11 months of age; Figure S4. Metabolic pathway enrichment maps of the liver of all offspring groups at 11 months of age; Table S1. R2Y and Q2Y of the pairwise OPLS-DA model; Table S2. Metabolites altered in the liver of GDM offspring at 11 months of age and modulating effects of n-3 PUFA.

Author Contributions: J.G. and D.L. conceived and designed the study; H.X. guided metabolomics data analysis; J.L., X.G. and W.C. provided help in laboratory work; J.G. analyzed data and drafted the initial manuscript; J.G. and D.L. critically reviewed the manuscript; all authors approved the final manuscripts as submitted.

Funding: This work was supported by the National Basic Research Program of China (973 Program: 2015CB553604); National Natural Science Foundation of China (NSFC: 81773433).

Conflicts of Interest: The authors have declared no conflict of interest.

\section{Abbreviations}

GDM, gestational diabetes mellitus; PUFA, polyunsaturated fatty acid; SOD, superoxide dismutase; MDA, malondialdehyde; GSH, glutathione; GSH-Px, glutathione peroxidase; CAT, catalase; TNF- $\alpha$, tumor necrosis factor- $\alpha$; IL-1 $\beta$, interleukin-1 $\beta$; IL-6, interleukin-6; IL-10, interleukin-10.

\section{References}

1. Pereira, T.J.; Moyce, B.L.; Kereliuk, S.M.; Dolinsky, V.W. Influence of maternal overnutrition and gestational diabetes on the programming of metabolic health outcomes in the offspring: Experimental evidence. Biochem. Cell Biol. 2015, 93, 438-451. [CrossRef] [PubMed]

2. Riediger, N.D.; Othman, R.A.; Suh, M.; Moghadasian, M.H. A Systemic review of the roles of n-3 Fatty Acids in Health and Disease. J. Am. Diet. Assoc. 2009, 109, 668-679. [CrossRef] [PubMed]

3. Nettleton, J.; Katz, R. N-3 long-chain polyunsaturated fatty acids in type 2 diabetes: A review. J. Am. Diet. Assoc. 2005, 105, 428-440. [CrossRef] [PubMed]

4. Poreba, M.; Mostowik, M.; Siniarski, A.; Golebiowska-Wiatrak, R.; Malinowski, K.P.; Haberka, M.; Konduracka, E.; Nessler, J.; Undas, A.; Gajos, G. Treatment with high-dose n-3 PUFAs has no effect on platelet function, coagulation, metabolic status or inflammation in patients with atherosclerosis and type 2 diabetes. Cardiovasc. Diabetol. 2017, 16, 50-61. [CrossRef] [PubMed]

5. Lindsay, R.S.; Westgate, J.A.; Beattie, J.; Pattison, N.S.; Gamble, G.; Mildenhall, L.F.J.; Breier, B.H.; Johnstone, F.D. Inverse changes in fetal insulin-like growth factor (IGF)-1 and IGF binding protein-1 in association with higher birth weight in maternal diabetes. Clin. Endocrinol. 2007, 66, 322-328. [CrossRef] [PubMed]

6. Yessoufou, A.; Soulaimann, N.; Merzouk, S.; Moutairou, K.; Ahissou, H.; Prost, H.; Simmoni, A.M.; Merzouk, A.; Khan, N.A. N-3 Fatty acids modulate antioxidant status in diabetic rats and their macrosomic offspring. Int. J. Obes. 2006, 30, 739-750. [CrossRef]

7. Pereira, T.J.; Fonseca, M.A.; Campbell, K.E.; Moyce, B.L.; Cole, L.K.; Hatch, G.M.; Doucette, C.A.; Klein, J.; Aliani, M.; Dolinsky, V.W. Maternal obesity characterized by gestational diabetes increases the susceptibility of rat offspring to hepatic steatosis via a disrupted liver metabolome. J. Physiol. 2015, 593, 3181-3197. [CrossRef]

8. Ruchat, S.; Hivert, M.; Bouchard, L. Epigenetic programming of obesity and diabetes by in utero exposure to gestational diabetes mellitus. Nutr. Rev. 2013, 71, S88-S94. [CrossRef]

9. Aubert, G.; Lansdorp, P.M. Telomeres and Aging. Physiol. Rev. 2008, 88, 557-579. [CrossRef]

10. Epel, E.S.; Blackburn, E.H.; Lin, J.; Dhabhar, F.S.; Adler, N.E.; Morrow, J.D.; Cawthon, R.M. Accelerated telomere shortening in response to life stress. Proc. Natl. Acad. Sci. USA 2004, 101, 17312-17315. [CrossRef] 
11. Blasco, M.A. Telomeres and human disease: Ageing, cancer and beyond. Nat. Rev. Genet. 2005, 6, 611-622. [CrossRef] [PubMed]

12. Zhao, H.; Li, H.; Chung, A.C.K.; Xiang, L.; Li, X.; Zheng, Y.; Luan, H.; Zhu, L.; Liu, W.; Peng, Y.; et al. Large-scale longitudinal metabolomics study reveals different trimester-specific alterations of metabolites in relation to gestational diabetes mellitus. J. Proteome Res. 2018, 18, 292-300. [CrossRef] [PubMed]

13. Li, J.; Huo, X.; Cao, Y.; Li, S.; Du, Z.; Shao, P.; Leng, J.; Zhang, C.; Sun, X.; Ma, R.C.W.; et al. Bile acid metabolites in early pregnancy and risk of gestational diabetes in Chinese women: A nested case-control study. EBioMedicine 2018, 35, 317-324. [CrossRef] [PubMed]

14. Gelaye, B.; Clish, C.B.; Denis, M.; Larrabure, G.; Tadesse, M.G.; Deik, A.; Pierce, K.; Bullock, K.; Dennis, C.; Enquobahrie, D.A.; et al. Metabolomics signatures associated with an oral glucose challenge in pregnant women. Diabetes Metab. 2018, 45, 39-46. [CrossRef] [PubMed]

15. O'Neill, K.; Alexander, J.; Azuma, R.; Xiao, R.; Snyder, N.; Mesaros, C.; Blair, I.; Pinney, S. Gestational diabetes alters the metabolomic profile in 2 nd trimester amniotic fluid in a sex-specific manner. Int. J. Mol. Sci. 2018, 19, 2696. [CrossRef] [PubMed]

16. Lu, Y.; Reichetzeder, C.; Prehn, C.; von Websky, K.; Slowinski, T.; Chen, Y.; Yin, L.; Kleuser, B.; Yang, X.; Adamski, J.; et al. Fetal serum metabolites are independently associated with gestational diabetes mellitus. Cell. Physiol. Biochem. 2018, 45, 625-638. [CrossRef]

17. Han, H.; Kang, G.; Kim, J.S.; Choi, B.H.; Koo, S. Regulation of glucose metabolism from a liver-centric perspective. Exp. Mol. Med. 2016, 48, e218. [CrossRef]

18. Barker, D.J.P. The origins of the developmental origins theory. J. Intern. Med. 2007, 261, 412-417. [CrossRef]

19. Hocher, B. More than genes: The advanced fetal programming hypothesis. J. Reprod. Immunol. 2014, 104, 8-11. [CrossRef]

20. Gauster, M.; Desoye, G.; Tötsch, M.; Hiden, U. The placenta and gestational diabetes mellitus. Curr. Diabetes Rep. 2012, 12, 16-23. [CrossRef]

21. Wang, K.; Jin, X.; Li, Q.; Sawaya, A.C.H.F.; Le Leu, R.K.; Conlon, M.A.; Wu, L.; Hu, F. Propolis from Different Geographic Origins Decreases Intestinal Inflammation and Bacteroides spp. Populations in a Model of DSS-Induced Colitis. Mol. Nutr. Food Res. 2018, 62, 80-91. [CrossRef] [PubMed]

22. Tian, X.; Liu, Y.; Ren, G.; Yin, L.; Liang, X.; Geng, T.; Dang, H.; An, R. Resveratrol limits diabetes-associated cognitive decline in rats by preventing oxidative stress and inflammation and modulating hippocampal structural synaptic plasticity. Brain Res. 2016, 1650, 1-9. [CrossRef] [PubMed]

23. Wei, Y.B.; Backlund, L.; Wegener, G.; Mathe, A.A.; Lavebratt, C. Telomerase dysregulation in the hippocampus of a rat model of depression: Normalization by lithium. Int. J. Neuropsychopharmacol. 2015, 18, 1-8. [CrossRef]

24. Kaijser, M.; EdstedtBonamy, A.K.; Akre, O.; Cnattingius, S.; Granath, F.; Norman, M.; Ekbom, A. Perinatal Risk Factors for Diabetes in Later Life. Diabetes 2009, 58, 523-526. [CrossRef] [PubMed]

25. Gastaldelli, A.; Cusi, K.; Pettiti, M.; Hardies, J.; Miyazaki, Y.; Berria, R.; Buzzigoli, E.; Sironi, A.M.; Cersosimo, E.; Ferrannini, E.; et al. Relationship Between Hepatic/Visceral Fat and Hepatic Insulin Resistance in Nondiabetic and Type 2 Diabetic Subjects. Gastroenterology 2007, 133, 496-506. [CrossRef] [PubMed]

26. Aref, A.M.; Ahmed, O.M.; Ali, L.A.; Semmler, M. Maternal rat diabetes mellitus deleteriously affects insulin sensitivity and beta-cell function in the offspring. J. Diabetes Res. 2013, 2013, 429154. [CrossRef] [PubMed]

27. Ding, G.; Wang, F.; Shu, J.; Tian, S.; Jiang, Y.; Zhang, D.; Wang, N.; Luo, Q.; Zhang, Y.; Jin, F.; et al. Transgenerational glucose intolerance with Igf2/H19 epigenetic alterations in mouse islet induced by intrauterine hyperglycemia. Diabetes 2012, 61, 1133-1142. [CrossRef] [PubMed]

28. Kamel, M.A.; Helmy, M.H.; Hanafi, M.Y.; Mahmoud, S.A.; Abo Elfetooh, H. Impaired peripheral glucose sensing in F1 offspring of diabetic pregnancy. J. Physiol. Biochem. 2014, 70, 685-699. [CrossRef]

29. Malcolm, J.C.; Lawson, M.L.; Gaboury, I.; Lough, G.; Keely, E. Glucose tolerance of offspring of mother with gestational diabetes mellitus in a low-risk population. Diabet. Med. 2006, 23, 565-570. [CrossRef]

30. Franks, P.W.; Looker, H.C.; Kobes, S.; Touger, L.; Tataranni, P.A.; Hanson, R.L.; Knowler, W.C. Gestational glucose tolerance and risk of type 2 diabetes in young Pima Indian offspring. Diabetes 2006, 55, 460-465. [CrossRef]

31. Dabelea, D.; Knowler, W.C.; Pettitt, D.J. Effect of diabetes in pregnancy on offspring: Follow-up research in the Pima Indians. J. Matern.-Fetal Med. 2000, 9, 83-88.

32. Moussa, S.A. Oxidative stress in diabetes mellitus. Romanian. J. Biophys. 2008, 18, 225-236. 
33. Ouassila, A.; Saad, S.; Meriem, K.; Amira, M.; Imen, M. The investigation of the oxidative stress-related parameters in type 2 diabetes mellitue. Can. J. Diabetes 2015, 39, 44-49.

34. Weitao, C.; Dandan, R.; Yuanhu, X.; Chao, N.; Youli, T.; Yang, W.; Kungao, Z.; Lu, C.; Litai, J.; Yi, T. Cardiac-specific overexpression of catalase prevents diabetes-induced pathological changes by inhibiting NF-kB signaling activation in the heart. J. Mol. Cell. Cardiol. 2015, 89, 314-325.

35. Akash, M.S.H.; Rehman, K.; Liaqat, A. Tumor necrosis factor-alpha: Role in development of insulin resistance and pathogenesis of type 2 diabetes mellitus. J. Cell. Biochem. 2018, 119, 105-110. [CrossRef]

36. Cai, D.; Yuan, M.; Frantz, D.F.; Melendez, P.A.; Hansen, L.; Lee, J.; Shoelson, S.E. Local and systemic insulin resistance resulting from hepatic activation of IKK-beta andNF-kappa B. Nat. Med. 2005, 11, 183-190. [CrossRef]

37. Vasconcelos-Moreno, M.P.; Fries, G.R.; Gubert, C.; dos Santos, B.T.M.Q.; Fijtman, A.; Sartori, J.; Ferrari, P.; Grun, L.K.; Parisi, M.M.; Guma, F.T.C.R.; et al. Telomere length, oxidative stress, inflammation and BDNF levels in siblings of patients with bipolar disorder: Implications for accelerated cellular aging. Int. J. Neuropsychopharmacol. 2017, 6, 445-454. [CrossRef]

38. Zhang, J.; Rane, G.; Dai, X.; Shanmugam, M.K.; Arfuso, F.; Samy, R.P.; Lai, M.K.P.; Kappei, D.; Kumar, A.P.; Sethi, G. Aging and the telomere connections: An intimate relationship with inflammation. Ageing Res. Rev. 2016, 25, 55-69. [CrossRef]

39. Xu, J.; Ye, J.; Wu, Y.; Zhang, H.; Luo, Q.; Han, C.; Ye, X.; Wang, H.; He, J.; Huang, H.; et al. Reduced fetal telomere length in gestational diabetes. PLoS ONE 2014, 9, e86161. [CrossRef]

40. Cross, J.A.; Brennan, C.; Gray, T.; Temple, R.C.; Dozio, N.; Hughes, J.C.; Levell, N.J.; Murphy, H.; Fowler, D.; Hughes, D.A.; et al. Absence of telomere shortening and oxidative DNA damage in the young adult offspring of women with pre-gestational type 1 diabetes. Diabetologia 2009, 52, 226-234. [CrossRef]

41. Gilfillan, C.; Naidu, P.; Gunawan, F.; Hassan, F.; Tian, P.; Elwood, N. Leukocyte telomere length in the neonatal offspring of mothers with gestational and pre-gestational diabetes. PLoS ONE 2016, 11, e163824. [CrossRef]

42. Chavez, J.A.; Summers, S.A. A Ceramide-centric view of insulin resistance. Cell Metab. 2012, 15, 585-594. [CrossRef]

43. Pardo, V.; González-Rodríguez, Á.; Muntané, J.; Kozma, S.C.; Valverde, Á.M. Role of hepatocyte S6K1 in palmitic acid-induced endoplasmic reticulum stress, lipotoxicity, insulin resistance and in oleic acid-induced protection. Food Chem. Toxicol. 2015, 80, 298-309. [CrossRef]

44. Boden, G.; Chen, X.; Stein, T.P. Gluconeogenesis in moderately and severely hyperglycemic patients with type 2 diabetes mellitus. Am. J. Physiol. Endocrinol. Metab. 2001, 280, E23-E30. [CrossRef]

45. De Weerth, C.; Zijl, R.H.; Buitelaar, J.K. Development of cortisol circadian rhythm in infancy. Early Hum. Dev. 2003, 73, 39-52. [CrossRef]

46. Phillips, D.I.; Barker, D.J.; Fall, C.H.; Seckl, J.R.; Whorwood, C.B.; Wood, P.J.; Walker, B.R. Elevated plasma cortisol concentrations: A link between low birth weight and the insulin resistance syndrome? J. Clin. Endocrinol. Metab. 1998, 83, 757-760.

47. Wu, J.H.; Micha, R.; Imamura, F.; Pan, A.; Biggs, M.L.; Ajaz, O.; Djousse, L.; Hu, F.B.; Mozaffarian, D. Omega-3 fatty acids and incident type 2 diabetes: A systematic review and meta-analysis. Br. J. Nutr. 2012, 107, S214-S227. [CrossRef]

48. Muramatsu, T.; Yatsuya, H.; Toyoshima, H.; Sasaki, S.; Li, Y.; Otsuka, R.; Wada, K.; Hotta, Y.; Mitsuhashi, H.; Matsushita, K. Higher dietary intake of alpha-linolenic acid is associated with lower insulin resistance in middle-aged Japanese. Prev. Med. 2010, 50, 272-276. [CrossRef]

49. Djoussé, L.; Biggs, M.L.; Lemaitre, R.N.; King, I.B.; Song, X.; Ix, J.H.; Mukamal, K.J.; Siscovick, D.S.; Mozaffarian, D. Plasma omega-3 fatty acids and incident diabetes in older adults. Am. J. Clin. Nutr. 2011, 94, 527-533. [CrossRef]

50. Behme, M.T. Nicotinamide and diabetes prevention. Nutr. Rev. 1995, 53, 137-139. [CrossRef]

51. Olmos, P.R.; Hodgson, M.I.; Maiz, A.; Manrique, M.; De Valdés, M.D.; Foncea, R.; Acosta, A.M.; Emmerich, M.V.; Velasco, S.; Muñiz, O.P.; et al. Nicotinamide protected first-phase insulin response (FPIR) and prevented clinical disease in first-degree relatives of type-1 diabetics. Diabetes Res. Clin. Pract. 2006, 71, 320-333. [CrossRef] [PubMed]

52. O'Brien, B.A.; Harmon, B.V.; Cameron, D.P.; Allan, D.J. Nicotinamide prevents the development of diabetes in the cyclophosphamide-induced NOD mouse model by reducing beta-cell apoptosis. J. Pathol. 2000, 191, 86-92. [CrossRef] 
53. Baliarsingh, S.; Beg, Z.H.; Ahmad, J. The therapeutic impacts of tocotrienols in type 2 diabetic patients with hyperlipidemia. Atherosclerosis 2005, 182, 367-374. [CrossRef] [PubMed]

54. Kuhad, A.; Bishnoi, M.; Tiwari, V.; Chopra, K. Suppression of NF- $\kappa \beta$ signaling pathway by tocotrienol can prevent diabetes associated cognitive deficits. Pharmacol. Biochem. Behav. 2009, 92, 251-259. [CrossRef] [PubMed]

55. Erdinest, N.; Shmueli, O.; Grossman, Y.; Ovadia, H.; Solomon, A. Anti-inflammatory effects of alpha linolenic acid on human corneal epithelial cells. Investig. Ophthalmol. Vis. Sci. 2012, 53, 4396-4406. [CrossRef]

56. Boon, J.; Hoy, A.J.; Stark, R.; Brown, R.D.; Meex, R.C.; Henstridge, D.C.; Schenk, S.; Meikle, P.J.; Horowitz, J.F.; Kingwell, B.A.; et al. Ceramides contained in LDL are elevated in type 2 diabetes and promote inflammation and skeletal muscle insulin resistance. Diabetes 2013, 62, 401-410. [CrossRef]

57. Berglund, T.; Wallström, A.; Nguyen, T.V.; Laurell, C.; Ohlsson, A.B. Nicotinamide: Antioxidative and DNA hypomethylation effects in plant cells. Plant Physiol. Biochem. 2017, 118, 551-560. [CrossRef]

58. Ying, W.; Min, Z. Nicotinamide improves sevoflurane-induced cognitive impairment through suppression of inflammation and anti-apoptosis in rat. Int. J. Clin. Exp. Med. 2015, 8, 20079-20085.

59. Abu-Bakar, A.; Hakkola, J.; Juvonen, R.; Rahnasto-Rilla, M.; Raunio, H.; A. Lang, M. Function and regulation of the Cyp2a5/CYP2A6 genes in response to toxic insults in the liver. Curr. Drug Metab. 2013, 14, 137-150. [CrossRef]

60. Skowrońska, M.; Albrecht, J. Alterations of blood brain barrier function in hyperammonemia: An overview. Neurotox. Res. 2012, 21, 236-244. [CrossRef]

(C) 2019 by the authors. Licensee MDPI, Basel, Switzerland. This article is an open access article distributed under the terms and conditions of the Creative Commons Attribution (CC BY) license (http://creativecommons.org/licenses/by/4.0/). 